\title{
Centralisers of spaces of symmetric tensor products and applications
}

\author{
Christopher Boyd \& Silvia Lassalle*
}

\begin{abstract}
We show that the centraliser of the space of $n$-fold symmetric injective tensors, $n \geq 2$, on a real Banach space is trivial. With a geometric condition on the set of extreme points of its dual, the space of integral polynomials we obtain the same result for complex Banach spaces. We give some applications of this results to centralisers of spaces of homogeneous polynomials and complex Banach spaces. In addition, we derive a Banach-Stone Theorem for spaces of vector-valued approximable polynomials.
\end{abstract}

\section{Introduction}

The notion of an M-ideal arose out of attempts to generalise the concept of a twosided ideal from a $\mathrm{C}^{*}$-algebra to a general Banach space setting. In the second part of their classical paper of 1971 Alfsen and Effros [1] further investigated the analogy by introducing the structure topology on the set of extreme points of the unit ball of $E^{\prime}$ as a generalisation of Jacobson's hull-kernel topology. It was in this context that they introduced the centraliser of a Banach space as the generalisation, to a Banach space setting, of the concept of the centre of a unital Banach algebra. However, Alfsen's and Effros's papers only consider real Banach spaces. To work with complex Banach spaces it was necessary to redefine the notation of centraliser, calling what is understood as the centraliser in the real case the multiplier algebra. This was first done by Behrends in [3].

This appears an opportune moment to introduce these concepts.

Definition 1 Let $E$ be a Banach space and $T: E \rightarrow E$ be a continuous linear operator. Then $T$ is a multiplier if every extreme point of the unit ball of $E^{\prime}$ is an eigenvalue of $T^{\prime}$. That is

$$
T^{\prime}(e)=a_{T}(e) e
$$

* This project was supported in part by Enterprise Ireland, International Collaboration Grant - 2004 (IC/2004/009).

*2000 Mathematics Subject Classification: Primary 46B20, 46G25; Secondary 46B04, 46B28. 
for every extreme point $e$ of the unit ball of $E^{\prime}$. We let $\operatorname{Mult}(E)$ denote the set of all multipliers on $E$.

Definition 2 Let $E$ be a Banach space. The centraliser of $E, Z(E)$, is the set of all $T \in \operatorname{Mult}(E)$ for which there is $S$ in $\operatorname{Mult}(E)$ with $S^{\prime}(e)=\overline{a_{T}(e)}$ e for all extreme points e of the unit ball of $E^{\prime}$.

In the above definition $\overline{a_{T}(e)}$ denotes the complex conjugate of $a_{T}(e)$.

The centraliser of any Banach space is a unital $\mathrm{C}^{*}$-algebra. When $E$ is a real Banach space the centraliser of $E$ coincides with the multiplier algebra of E.

We say that $Z(E)$ is trivial if $Z(E)=\mathbf{K}$.Id, $(\mathbf{K}=\mathbf{R}$ or $\mathbf{C}$ depending on whether $E$ is a real or complex Banach space).

Let $E$ and $F$ be Banach spaces and $u$ be an element of the tensor product $E \otimes F$. We define the injective norm of $u$ as

$$
\|u\|_{\epsilon}=\sup \left\{\left|\sum_{i=1}^{k} \phi\left(x_{i}\right) \psi\left(y_{i}\right)\right|: u=\sum_{i=1}^{k} x_{i} \otimes y_{i}: \phi \in B_{E^{\prime}}, \psi \in B_{F^{\prime}}\right\} .
$$

We shall use $E \bigotimes_{\epsilon} F$ to denote $E \otimes F$ endowed with the injective norm and $E \widehat{\bigotimes}_{\epsilon} F$ to denote the completion of $E \bigotimes_{\epsilon} F$ with respect the norm.

Given a Banach space $E$ we can form the space $\bigotimes_{n} E$ of all $n$-fold tensors in $E$. We consider the subspace, $\bigotimes_{n, s} E$, of $\bigotimes_{n} E$ consisting of all tensors of the form $\sum_{i=1}^{k} \lambda_{i} x_{i} \otimes x_{i} \otimes \ldots \otimes x_{i}$, where $\lambda_{i}= \pm 1$. Such $n$-fold tensors are said to be symmetric. Given an $n$-fold symmetric tensor $\sum_{i=1}^{k} \lambda_{i} x_{i} \otimes x_{i} \otimes \ldots \otimes x_{i}$ on $E$ we define its injective norm as

$$
\sup _{\phi \in B_{E^{\prime}}}\left|\sum_{i=1}^{k} \lambda_{i} \phi\left(x_{i}\right)^{n}\right|
$$

This may also be regarded as the norm inherited from $\mathcal{P}\left({ }^{n} E^{\prime}\right)$, the space of all $n$-homogeneous polynomials on $E^{\prime}$. We denote the completion of $\bigotimes_{n, s} E$ with respect to this norm by $\widehat{\bigotimes}_{n, s, \epsilon} E$.

In [24] Wickstead shows that the centraliser of the completed injective tensor product of the Banach spaces $E$ and $F$ is the completion of $Z(E) \otimes Z(F)$ with respect to the strong operator topology. If $E$ and $F$ are dual spaces then we have that $Z\left(E \widehat{\bigotimes}_{\epsilon} F\right)=Z(E) \widehat{\bigotimes}_{\epsilon} Z(F)$.

In [23] Wend Werner gives an alternative description of $Z\left(E \widehat{\bigotimes}_{\epsilon} F\right)$ as a space of continuous bounded functions using the set of extreme points of the unit balls of $E^{\prime}$ and $F^{\prime}$. To be more specific, given a Banach space $E$ define $Z_{E}$ by $Z_{E}=\overline{\operatorname{Ext}_{\mathbf{R}}\left(B_{E^{\prime}}\right)} w^{*} \backslash\{0\}$. Endow $Z_{E}$ with the weak ${ }^{*}$-topology. For each multiplier $T$ on $E$ the function $a_{T}$ extends, by weak*-continuity, to $Z_{E}$, see [14, Lemma 3.2]. An equivalence relation $\cong$ is defined on $Z_{E}$ by $p \cong q$ if $a_{T}(p)=a_{T}(q)$ for all $T$ in $Z(E)$. The space $\Theta_{E}$ is defined as $Z_{E} / \cong$. Given 
Hausdorff topological spaces $R$ and $S$ let $R \times{ }_{k} S$ denote the product of $R$ and $S$ endowed with the finest topology which coincides with the product topology on compact sets. Werner [23] shows that the centraliser of $E \widehat{\bigotimes}_{\epsilon} F$ can be identified with $C^{b}\left(\Theta_{E} \times_{k} \Theta_{F}\right)$, the space of continuous bounded functions on $\Theta_{E} \times_{k} \Theta_{F}$.

In this paper we will show that the situation for symmetric injective tensor products is very different. For further information on centralisers we refer the reader to [1], [3] and [14].

\section{Centraliser of symmetric tensor products of real Banach spaces}

An extreme point of the (closed) unit ball of $E, \bar{B}_{E}$, is a point $x$ with the property that whenever $x=\lambda y+(1-\lambda) z$ for $y, z$ in $\bar{B}_{E}$ and $0<\lambda<1$, then, $x=y=z$. We shall use $\operatorname{Ext}_{\mathbf{R}}(E)$ to denote the set of extreme points of $\bar{B}_{E}$.

We recall some notation and definitions for the theory of homogeneous polynomials on Banach spaces.

Let $E$ and $F$ be Banach spaces. A function $P: E \rightarrow F$ is said to be a (continuous) $n$-homogeneous polynomial if there is a (continuous) $n$-linear map $L_{P}: \underbrace{E \times \ldots \times E}_{\text {n-times }} \rightarrow F$ such that $P(x)=L_{P}(x, \ldots, x)$ for all $x \in E$. Continuous $n$-homogeneous polynomials are bounded on the unit ball. We denote by $\mathcal{P}\left({ }^{n} E ; F\right)$ the Banach space of all continuous $n$-homogeneous polynomials from $E$ into $F$ endowed with the norm $\|P\|:=\sup _{\|x\| \leq 1}\|P(x)\|_{F}$.

An $n$-homogeneous polynomial $P \in \mathcal{P}\left({ }^{n} E ; F\right)$ is said to be of finite type if there are $\left\{\phi_{j}\right\}_{j=1}^{k}$ in $E^{\prime}$ and $\left\{y_{j}\right\}_{j=1}^{k}$ in $F$ such that $P(x)=\sum_{j=1}^{k} \phi_{j}(x)^{n} y_{j}$ for all $x$ in $E$. Polynomials in the closure of the finite type $n$-homogeneous polynomials in $\mathcal{P}\left({ }^{n} E ; F\right)$ are called the approximable polynomials. We use $\mathcal{P}_{f}\left({ }^{n} E ; F\right)$ to denote the space of finite type $n$-homogeneous polynomials and $\mathcal{P}_{A}\left({ }^{n} E ; F\right)$ to denote the space of all $n$-homogeneous approximable polynomials. When $F$ is $\mathbf{R}$ or $\mathbf{C}$ we omit $F$ and write $\mathcal{P}\left({ }^{n} E\right)$ or $\mathcal{P}_{A}\left({ }^{n} E\right)$, for example. We refer to [10] for further information on polynomials on Banach spaces.

A scalar-valued polynomial $P$ on $E$ is said to be integral if there is a regular Borel measure $\mu$ on $\left(B_{E^{\prime}}, \sigma\left(E^{\prime}, E\right)\right)$ such that

$$
P(x)=\int_{B_{E^{\prime}}} \phi(x)^{n} d \mu(\phi)
$$

for every $x$ in $E$. We write $\mathcal{P}_{I}\left({ }^{n} E\right)$ for the space of all $n$-homogeneous integral polynomials on $E$. We define the integral norm of an integral polynomial $P$, $\|P\|_{I}$, as the infimum of $\|\mu\|$ taken over all regular Borel measures which satisfy (1). It is shown in [11] that the dual of $\widehat{\bigotimes}_{n, s, \epsilon} E$ is isometrically isomorphic to $\left(\mathcal{P}_{I}\left({ }^{n} E\right),\|\cdot\|_{I}\right)$.

Let $E$ be a real or complex Banach space and $n$ be a positive integer. It is shown in [6, Proposition 1] that the set of (real) extreme points of the unit ball 
of $\mathcal{P}_{I}\left({ }^{n} E\right)$ is contained in $\left\{ \pm \varphi^{n}: \varphi \in X^{\prime},\|\varphi\|=1\right\}$. Hence, given a Banach space $E$ and a positive integer $n$ we use $\mathcal{E}_{n}\left(E^{\prime}\right)$ to denote the set

$$
\left\{\varphi \in E^{\prime}: \varphi^{n} \text { is an extreme point of } \bar{B}_{\mathcal{P}_{I}\left({ }^{n} E\right)}\right\} .
$$

Theorem 3 Let $E$ be a real Banach space and $n$ be a positive integer which is greater than or equal to 2. Then $Z\left(\widehat{\bigotimes}_{n, s, \epsilon} E\right)$ is trivial.

Proof: First, we assume that the dimension of $E$ is at least 3. Let us suppose that $T: \widehat{\bigotimes}_{n, s, \epsilon} E \rightarrow \widehat{\bigotimes}_{n, s, \epsilon} E$ is a multiplier. We assume that $T$ is not identically zero. Then we can find $a: \mathcal{E}_{n}\left(E^{\prime}\right) \rightarrow \mathbf{R}$ such that

$$
T^{\prime}\left(\varphi^{n}\right)=a(\varphi) \varphi^{n}
$$

for all $\varphi \in \mathcal{E}_{n}\left(E^{\prime}\right)$.

The function $a$ can be continuously extended to $E^{\prime} \backslash\{0\}$ as follows:

If $T^{\prime}\left(\varphi^{n}\right)=0$ set $a(\varphi)=0$.

Now suppose that $\|\varphi\|=1$ and $T^{\prime}\left(\varphi^{n}\right) \neq 0$. By the Bishop-Phelps Theorem and [6, Proposition 5] we can find a sequence $\left(\varphi_{k}\right)_{k}$ in $\mathcal{E}_{n}\left(E^{\prime}\right)$ with $\left\|\varphi_{k}\right\|=1$ so that $\varphi_{k} \rightarrow \varphi$. By continuity of $T^{\prime}$ we can assume that $a\left(\varphi_{k}\right) \neq 0$.

Since $T^{\prime}\left(\varphi_{k}^{n}\right)$ converges to $T^{\prime}\left(\varphi^{n}\right)$ we have that

$$
\left\|T^{\prime}\left(\varphi_{k}^{n}\right)\right\|=\left|a\left(\varphi_{k}\right)\right|\left\|\varphi_{k}^{n}\right\|=\left|a\left(\varphi_{k}\right)\right|
$$

converges to $\left\|T^{\prime}\left(\varphi^{n}\right)\right\|$. Thus the limit of $\left|a\left(\varphi_{k}\right)\right|$ exists and is equal to $\left\|T^{\prime}\left(\varphi^{n}\right)\right\|$. Since $|a(\psi)|$ is bounded by $\|T\|$, the sequence $\left(a\left(\varphi_{k}\right)\right)_{k}$ is Cauchy. It follows that $\lim _{k \rightarrow \infty} a\left(\varphi_{k}\right)= \pm\left\|T^{\prime}\left(\varphi^{n}\right)\right\|$. Therefore, as $\lim _{k \rightarrow \infty} a\left(\varphi_{k}\right) \neq 0$, we may assume that the sign of each term in the sequence $\left(a\left(\varphi_{k}\right)\right)_{k}$ is constant. Suppose that $a\left(\varphi_{k}\right)>0$ for all $k$. If $\left(\psi_{k}\right)_{k}$ is any other sequence in $\mathcal{E}_{n}\left(E^{\prime}\right)$ which converges to $\varphi$ then we can assume that the sign of each $a\left(\psi_{k}\right)$ must also be positive. In this case we define $a(\varphi)$ to be $\left\|T^{\prime}\left(\varphi^{n}\right)\right\|$. Otherwise, $a(\varphi)$ will be $-\left\|T^{\prime}\left(\varphi^{n}\right)\right\|$.

Let us show that $a$ is continuous at $\varphi$. To see this we consider the case when $a(\varphi)>0$. We claim there is $\delta>0$ so that $a(\psi)>0$ for $\|\varphi-\psi\|<\delta$. If not, we can find a sequence $\left(\psi_{k}\right)_{k}$ in $\mathcal{E}_{n}\left(E^{\prime}\right)$ such that $\lim _{k \rightarrow \infty} \psi_{k}=\varphi$ and $a\left(\psi_{k}\right)<0$. By the above this is impossible and therefore $a(\psi)=\left\|T^{\prime}\left(\psi^{n}\right)\right\|$ for $\psi$ sufficiently close to $\varphi$. Hence $a$ is continuous at $\varphi$. An analogous proof shows that $a$ is continuous at $\varphi$ when $a(\varphi)<0$ or $a(\varphi)=0$.

By homogeneity we have that

$$
T^{\prime}\left(\varphi^{n}\right)=a(\varphi /\|\varphi\|) \varphi^{n}
$$

for all $\varphi \in E^{\prime} \backslash\{0\}$ and therefore by defining $a(\varphi)$ to be $a(\varphi /\|\varphi\|)$ for $\varphi \in E^{\prime} \backslash\{0\}$ we get a continuous function $a: E^{\prime} \backslash\{0\} \rightarrow \mathbf{R}$ such that

$$
T^{\prime}\left(\varphi^{n}\right)=a(\varphi) \varphi^{n}
$$

for all $\varphi \in E^{\prime} \backslash\{0\}$. Thus $T^{\prime}\left(\varphi^{n}\right)=a(\varphi) \varphi^{n}$ for all $\varphi$ in $E^{\prime}$ with $\|\varphi\|=1$. 
In order to show that $a$ is constant on $E^{\prime} \backslash\{0\}$ we consider $\left(T^{\prime}\right)^{2}$. Then

$$
\left(T^{\prime}\right)^{2}\left(\varphi^{n}\right)=a(\varphi)^{2} \varphi^{n}
$$

for all $\varphi \in E^{\prime} \backslash\{0\}$.

Letting $b(\varphi)=\left(a(\varphi)^{2}\right)^{1 / n}$ (we take the positive $n$th real root) we get that

$$
\left(T^{\prime}\right)^{2}\left(\varphi^{n}\right)=(b(\varphi) \varphi)^{n}
$$

for all $\varphi$ in $E^{\prime} \backslash\{0\}$. Fix $\xi, \psi$ in the unit sphere of $E^{\prime}$ for which $\xi^{n}$ and $\psi^{n}$ are linearly independent and for which $a(\xi) \neq 0$ and $a(\psi) \neq 0$. This is always possible since $a$ is continuous and for $\psi$ sufficiently close to $\xi$ we have that $\xi^{n}$ and $\psi^{n}$ are linearly independent. Consider $\eta$ in $E^{\prime}$ so that $\xi, \psi$ and $\eta$ are linearly independent. Let $V$ denote the subspace of $E^{\prime}$ spanned by $\xi, \psi$ and $\eta$. By [22, Exercise 10.5.5] there is a continuous linear mapping $r: V \rightarrow V$ such that

$$
\left(T^{\prime}\right)^{2}\left(\varphi^{n}\right)=(r(\varphi))^{n}
$$

for all $\varphi \in V$. (We need $\psi$ to avoid the degenerate rank 1 case.) By multiplication by -1 if necessary we may assume that $r(\xi)=b(\xi) \xi$. Taking $n^{\text {th }}$ roots we see that $r(\eta)= \pm b(\eta) \eta$. Suppose that $r(\eta)=-b(\eta) \eta$. Now suppose that $r(\xi+\eta)=b(\xi+\eta)(\xi+\eta)$. (A similar argument works if $r(\xi+\eta)=-b(\xi+\eta)(\xi+\eta)$ ). By linearity of $r$ we have that

$$
b(\xi+\eta)(\xi+\eta)=b(\xi) \xi-b(\eta) \eta .
$$

This gives us that

$$
(b(\xi+\eta)-b(\xi)) \xi=-(b(\eta)+b(\xi+\eta)) \eta .
$$

As $\xi$ and $\eta$ are linearly independent we get that

$$
b(\xi)=b(\xi+\eta)=-b(\eta)
$$

which is impossible as $b(\xi)$ is non-zero and $b(\eta)$ is nonnegative. Therefore we have that $r(\varphi)=b(\varphi) \varphi$ for all $\varphi \in V$. As $r$ is linear we get

$$
b(\xi+\eta)(\xi+\eta)=b(\xi) \xi+b(\eta) \eta .
$$

This gives us that

$$
(b(\xi+\eta)-b(\xi)) \xi=(b(\eta)-b(\xi+\eta)) \eta .
$$

As $\xi$ and $\eta$ are linearly independent we get that

$$
b(\xi)=b(\xi+\eta)=b(\eta)
$$

and $b$ is constant on $V$. As the only constraint on $\eta$ is that $\xi, \psi$ and $\eta$ are linearly independent $b$ must be constant on $E$. Let $U=\left\{\varphi \in E^{\prime} \backslash\{0\}: a(\varphi)=\sqrt{b}\right\}$ and $V=\left\{\varphi \in E^{\prime} \backslash\{0\}: a(\varphi)=-\sqrt{b}\right\}$. As $U \cup V=E^{\prime} \backslash\{0\}, U \cap V=\emptyset$ and $U$ and $V$ 
are both closed it follows that either $U$ or $V$ is empty and therefore $a$ is constant. Thus $T^{\prime}\left(\varphi^{n}\right)=a \varphi^{n}$ for $\varphi \in E^{\prime}$. We now claim that $T=a I_{\widehat{\bigotimes}_{n, s, \epsilon} E}$. Suppose that this is not true. As elements of the form $x^{n}, x \in E$, span a dense subspace of $\widehat{\bigotimes}_{n, s, \epsilon} E$ we can assume that there is $x$ in $E$ so that $T\left(x^{n}\right)=\theta \neq a x^{n}$. Choose $\varphi \in E^{\prime}$ so that $\left\langle\theta, \varphi^{n}\right\rangle \neq a \varphi(x)^{n}$. Then we have

$$
\left\langle T\left(x^{n}\right), \varphi^{n}\right\rangle \neq\left\langle a x^{n}, \varphi^{n}\right\rangle
$$

or

$$
\left\langle x^{n}, T^{\prime}\left(\varphi^{n}\right)\right\rangle \neq\left\langle x^{n}, a \varphi^{n}\right\rangle .
$$

This contradicts the fact that $T^{\prime}\left(\varphi^{n}\right)=a \varphi^{n}$ for all $\varphi \in E^{\prime}$ and therefore $T=$ $a \operatorname{Id}_{\widehat{\otimes}_{n, s, \epsilon} E}$ as claimed. Hence, it follows that $\widehat{\bigotimes}_{n, s, \epsilon} E$ has trivial centraliser.

When the $\operatorname{dim} E=2$ the above argument can be easily adapted to get the desired result.

Since $\widehat{\bigotimes}_{n, s, \epsilon} E^{\prime}$ is isometrically isomorphic to $\mathcal{P}_{A}\left({ }^{n} E\right)$ (see [10]) we obtain:

Corollary 4 Let $E$ be a real Banach space and $n$ be a positive integer which is at least 2. Then $Z\left(\mathcal{P}_{A}\left({ }^{n} E\right)\right)$ is trivial.

In [5] we studied the (surjective) isometries of spaces of scalar-valued homogeneous polynomials. We showed that if $E$ and $F$ are real Banach spaces, $n$ is a positive integer and $T$ is an isometric isomorphism from $\mathcal{P}_{A}\left({ }^{n} E\right)$ onto $\mathcal{P}_{A}\left({ }^{n} F\right)$ then there is an isometric isomorphism $s: E^{\prime} \rightarrow F^{\prime}$ such that $T(P)= \pm \bar{P} \circ s^{\prime} \circ J_{F}$ for all $P \in \mathcal{P}_{A}\left({ }^{n} E\right)$. Here $J_{F}$ is the canonical embedding of $F$ into $F^{\prime \prime}$ and $\bar{P}$ is the Aron-Berner extension of $P$ from $E$ to $E^{\prime \prime}$ (see [2]). Using [19], it follows that $\mathcal{P}_{A}\left({ }^{n} E\right)$ is isometrically isomorphic to $\mathcal{P}_{A}\left({ }^{n} F\right)$ if and only if $E^{\prime}$ is isometrically isomorphic to $F^{\prime}$. This characterisation of the isometries of spaces of approximable polynomials extends to complex Banach space with an additional geometric assumption. Our work to date on the centraliser of the space of symmetric injective tensor products allows us to extend the results in Section 3 of [5] to the vector-valued case. We will use the fact that given Banach spaces $E$ and $F$ every $P$ in $\mathcal{P}\left({ }^{n} E ; F\right)$ has a norm-preserving extension to $\bar{P}$ in $\mathcal{P}\left({ }^{n} E^{\prime \prime} ; F^{\prime \prime}\right)$, see [2].

Theorem 5 Let $E$ be a real Banach space such that $E^{\prime}$ has the approximation property, $n$ be a positive integer which is greater than or equal to 2 and $F$ be a smooth reflexive real Banach space. Let $T: \mathcal{P}_{A}\left({ }^{n} E ; F\right) \rightarrow \mathcal{P}_{A}\left({ }^{n} E ; F\right)$ be an isometry. Then there are isometries $s: E^{\prime} \rightarrow E^{\prime}$ and $S: F \rightarrow F$ such that

$$
T(P)=S\left(\bar{P} \circ s^{\prime} \circ J_{E}\right)
$$

for all $P \in \mathcal{P}_{A}\left({ }^{n} E ; F\right)$.

Proof: We assume that the dimension of $F$ is at least 2. First we note that from the remark following $[4$, Theorem 4$]$ we get that $\mathcal{P}_{A}\left({ }^{n} E\right)$ has the approximation property and therefore

$$
\mathcal{P}_{A}\left({ }^{n} E ; F\right)=\mathcal{P}_{A}\left({ }^{n} E\right) \widehat{\bigotimes}_{\epsilon} F
$$


holds isometrically.

It follows from Corollary 4 and the observation following [16, Theorem 3] that $T$ has one of the two following forms

(a) There is a Banach space $Z$ so that $\mathcal{P}_{A}\left({ }^{n} E\right)$ is isometrically isomorphic to $Z \widehat{\otimes}_{\epsilon} F$ and

$$
T(z \otimes h \otimes k)=z \otimes k \otimes h
$$

for all $z \in Z$ and $h, k \in F$.

(b) There are isometries $T_{1}$ of $\mathcal{P}_{A}\left({ }^{n} E\right)$ and $S$ of $F$ such that

$$
T(P \otimes y)=T_{1}(P) \otimes S(y)
$$

for all $P \in \mathcal{P}_{A}\left({ }^{n} E\right)$ and $y \in F$.

Suppose (a) occurs. We know from [21, Theorem 1.1] that

$$
\operatorname{Ext}_{\mathbf{R}}\left(\mathcal{P}_{I}\left({ }^{n} E^{\prime}\right)\right)=\operatorname{Ext}_{\mathbf{R}}\left(Z^{\prime}\right) \bigotimes S_{F^{\prime}}
$$

Fix $z^{\prime} \in \operatorname{Ext}_{\mathbf{R}}\left(Z^{\prime}\right)$ and consider the set $\left\{z^{\prime} \otimes y^{\prime}: y^{\prime} \in F^{\prime},\left\|y^{\prime}\right\|=1\right\}$. Then, by [6, Proposition 1] there is a subset $U$ of the unit sphere of $E^{\prime \prime}$ such that $\left\{z^{\prime} \otimes y^{\prime}\right.$ : $\left.y^{\prime} \in F^{\prime},\left\|y^{\prime}\right\|=1\right\}$ can be identified with the set $\left\{ \pm x^{n}: x \in U\right\}$. Thus the set of all scalar multiples of $\left\{ \pm x^{n}: x \in U\right\}$ is isomorphic to $z^{\prime} \otimes F^{\prime}$. This means that the set $z^{\prime} \otimes F^{\prime}$ cannot be a vector space unless $U$ is a singleton in which case the dimensions of both sides differ. Therefore we have a contradiction. The first possibility is ruled out and hence $T$ must have the form given in (b). From [5, Theorem 7] it follows that each isometry $T_{1}$ of $\mathcal{P}_{A}\left({ }^{n} E\right)$ has the form

$$
T_{1}(P)= \pm \bar{P} \circ s^{\prime} \circ J_{E}
$$

for some isometric isomorphism $s$ of $E^{\prime}$ and the result follows.

We conclude this section by showing how some of the properties of $\widehat{\bigotimes}_{n, s, \epsilon} E$ are determined by its centraliser.

Definition 6 A Banach space $E$ has the strong Banach-Stone property if given locally compact Hausdorff spaces $K$ and $L$ and an isometric isomorphism $T$ of $C_{o}(K ; E)$ onto $C_{o}(L ; E)$ there is a homeomorphism $\phi: L \rightarrow K$ and a continuous function $h$ from $L$ into the isometries of $E$ endowed with the strong operator topology such that

$$
(T(f))(x)=h(x) f(\phi(x))
$$

for all $f \in C_{o}(K, E)$ and all $x \in L$.

We have:

Proposition 7 Let $E$ be a real Banach space and $n$ be a positive integer which is at least 2. Then $\mathcal{P}_{A}\left({ }^{n} E\right)$ and $\widehat{\bigotimes}_{n, s, \epsilon} E$ have the strong Banach-Stone property. 
Proof: Since both $\widehat{\bigotimes}_{n, s, \epsilon} E$ and $\mathcal{P}_{A}\left({ }^{n} E\right)$ has trivial centraliser it follows from $[3$, Theorem 8.11$]$ that both satisfy the strong Banach-Stone property.

Definition $8 \mathrm{~A}$ Banach space $E$ is a $C_{\sigma}$-space if there is a compact Hausdorff set $K$ and an involutory homeomorphism $\sigma: K \rightarrow K\left(\sigma^{2}=I d\right)$ such that $E$ is isometrically isomorphic to

$$
\{f \in C(K): f(x)=-f(\sigma(x)) \text { for all } x \in K\} .
$$

From Theorem 3 and [14, Theorem II.5.9] we get:

Proposition 9 Let $E$ be a real Banach space and $n$ be a positive integer which is at least 2. Then $\widehat{\bigotimes}_{n, s, \epsilon} E$ is not a $C_{\sigma}$-space.

\section{The centraliser of $\mathcal{P}_{A}\left({ }^{n} E\right)$ when $E$ is complex}

Let us turn our attention to the complex case. We shall see that things are not as straightforward here.

Let $E$ be a complex Banach space. We shall use $\mathcal{P}_{f^{*}}\left({ }^{n} E^{\prime}\right)$ to denote all $n$-homogeneous polynomials $P$ on $E^{\prime}$ of the form

$$
\sum_{j=1}^{k} x_{j}^{n}
$$

where $x_{j} \in E$. The closure of $\mathcal{P}_{f^{*}}\left({ }^{n} E^{\prime}\right)$ in $\mathcal{P}\left({ }^{n} E^{\prime}\right)$ is denoted by $\mathcal{P}_{A^{*}}\left({ }^{n} E^{\prime}\right)$ and is called the spaces of weak* approximable $n$-homogeneous polynomials. The space $\mathcal{P}_{A^{*}}\left({ }^{n} E^{\prime}\right)$ is isometrically isomorphic to $\widehat{\bigotimes}_{n, s, \epsilon} E$.

The following terminology was introduced in [5].

Definition 10 An operator $T: \mathcal{P}_{A}\left({ }^{n} E\right) \rightarrow \mathcal{P}_{A}\left({ }^{n} F\right)$ is said to be power-preserving or a power-preserver if for all $\varphi \in E^{\prime}$ we have $T\left(\varphi^{n}\right)=\psi^{n}$ for some $\psi$ in $F^{\prime}$.

Theorem 11 Let $E$ be a complex Banach space and $n$ be a positive integer which is greater than or equal to 2. Suppose that $\overline{\mathcal{E}_{n}\left(E^{\prime}\right)} w^{*}=\overline{\mathcal{E}_{2 n}\left(E^{\prime}\right)} w^{*}$. Then the centraliser of $\widehat{\bigotimes}_{n, s, \epsilon} E$ is trivial.

Proof: First we assume that $\operatorname{dim} E \geq 3$. Take $T \not \equiv 0$ in $\mathrm{Z}\left(\widehat{\bigotimes}_{n, s, \epsilon} E\right)$. Then $T^{\prime}$ can be considered as a linear operator from $\mathcal{P}_{I}\left({ }^{n} E\right)$ into $\mathcal{P}_{I}\left({ }^{n} E\right)$. Let $i: E^{\prime} \rightarrow$ $\mathcal{P}_{I}\left({ }^{n} E\right)$ denote the $n$-homogeneous polynomial $i(\varphi)=\varphi^{n}$. Given $x$ and $y$ in $E$ and $0 \leq k \leq n$, the Borel Transform tells us that $x^{k} y^{n-k}$ may be regarded as a continuous linear functional on $\mathcal{P}_{I}\left({ }^{n} E\right)$ with $x^{k} y^{n-k}\left(\varphi^{n}\right)=\varphi^{k}(x) \varphi^{n-k}(y)$ for all $\varphi \in E^{\prime}$. Hence for any $x, y$ in $E$ the function $x^{k} y^{n-k} \circ T^{\prime} \circ i \in \mathcal{P}_{A^{*}}\left({ }^{n} E^{\prime}\right)=$ $\widehat{\bigotimes}_{n, s, \epsilon} E$. Fix $x$ in $E$ and consider $y \in E$ which is non-zero and not a linear 
multiple of $x$. Let us use $Q_{k}$ to denote the $n$-homogeneous polynomial $x^{n-k} y^{k} \circ$ $T^{\prime} \circ i$. For $\varphi \in \mathcal{E}_{n}\left(E^{\prime}\right)$ we have that

$$
Q_{0}(\varphi) / Q_{1}(\varphi)=Q_{1}(\varphi) / Q_{2}(\varphi)=\ldots=Q_{n-1}(\varphi) / Q_{n}(\varphi)=\varphi(x) / \varphi(y) .
$$

Since each of the $Q_{k}$ 's is weak*-continuous this implies that

$$
Q_{k}(\varphi) Q_{k+2}(\varphi)=Q_{k+1}(\varphi)^{2}
$$

for all $\varphi \in{\overline{\mathcal{E}_{n}\left(E^{\prime}\right)}}^{w^{*}}={\overline{\mathcal{E}_{2 n}\left(E^{\prime}\right)}}^{w^{*}}$. As the extreme points of $\mathcal{P}_{I}\left({ }^{2 n} E\right)$ separate $\widehat{\bigotimes}_{2 n, s, \epsilon} E$, [15, Corollary II.13.3], we have that

$$
Q_{k}(\varphi) Q_{k+2}(\varphi)=Q_{k+1}(\varphi)^{2}
$$

for all $\varphi \in E^{\prime}$. Hence we get that

$$
Q_{0}(\varphi) / Q_{1}(\varphi)=Q_{1}(\varphi) / Q_{2}(\varphi)=\ldots=Q_{n-1}(\varphi) / Q_{n}(\varphi)
$$

for all $\varphi \in E^{\prime}$. We write this common ratio in lowest possible form as $R(\varphi) / S(\varphi)$. We have that $\operatorname{deg} R=\operatorname{deg} S=p$. As $y$ is not a linear multiple of $x$ we have that $p>0$. However, as

$$
Q_{0}(\varphi) / Q_{n}(\varphi)=\left(Q_{0} / Q_{1}\right)(\varphi)\left(Q_{1} / Q_{2}\right)(\varphi) \ldots\left(Q_{n-1} / Q_{n}\right)(\varphi)=(R(\varphi) / S(\varphi))^{n},
$$

we have that $p \leq 1$ and so both $R$ and $S$ belong to $E^{\prime \prime}$. Hence there is $\lambda \in \mathbf{C}$ so that $Q_{k}(\varphi)=\lambda R(\varphi)^{n-k} S(\varphi)^{k}$.

In particular,

$$
\left\langle\varphi^{n}, \lambda R^{n}\right\rangle=Q_{0}(\varphi)=\left\langle T^{\prime}\left(\varphi^{n}\right), x^{n}\right\rangle=\left\langle\varphi^{n}, T\left(x^{n}\right)\right\rangle
$$

for all $\varphi$ in $E^{\prime}$ for some constant $\lambda$. Further, if $\left(\varphi_{\alpha}\right)_{\alpha}$ is a weak*-null net in $E^{\prime}$ then $R\left(\varphi_{\alpha}\right)$ is null and therefore $R$ may be regarded as an element of $E$.

By [5, Proposition 14] it follows that $T$ is a power-preserver.

We claim that $T\left(x^{n}\right)$ is a scalar multiple of $x^{n}$ for each $x \in E$. Suppose, that this is not true. Then we can find $x$ and $y$ linearly independent in $E$ so that $T\left(x^{n}\right)=y^{n}$. Since the centraliser of $\widehat{\bigotimes}_{n, s, \epsilon} E$ is a vector space $I+T$ is also in the centraliser and hence also is a power-preserver. As $x^{n}$ and $y^{n}$ are linearly independent $(I+T)\left(x^{n}\right)=z^{n}$ with $x, y$ and $z$ linearly independent. Choose $\varphi$ and $\psi$ in $E^{\prime}$ so that $\varphi(x)=\psi(y)=0$ and $\varphi(z)=\psi(z)=1$. Then we

$$
\left\langle(I+T)\left(x^{n}\right), \varphi^{n-1} \psi\right\rangle=\left\langle x^{n}+y^{n}, \varphi^{n-1} \psi\right\rangle=0
$$

while

$$
\left\langle z^{n}, \varphi^{n-1} \psi\right\rangle=1
$$

a contradiction and therefore $T\left(x^{n}\right)$ is a linear multiple of $x^{n}$ for each $x \in E$. Let use $\lambda_{x}$ to denote the eigenvalue of $x^{n}$.

Given $\varphi \in \mathcal{E}_{n}\left(E^{\prime}\right)$ we shall use $a(\varphi)$ to denote the eigenvalue of $\varphi^{n}$ under the operator $T^{\prime}$. Since $T$ is in the centraliser of $\widehat{\bigotimes}_{n, s, \epsilon} E$ there is a multiplier $S$ so 
that $S^{\prime}\left(\varphi^{n}\right)=\overline{a(\varphi)} \varphi^{n}$ for all $\varphi \in \mathcal{E}_{n}\left(E^{\prime}\right)$. In order to show that $T$ is a multiple of the identity we will prove that both $S+T$ and $S-T$ are multiples of the identity. Fix $x$ in $E$ with $\|x\|=1$. We can choose $\varphi \in \mathcal{E}_{n}\left(E^{\prime}\right)$ so that $\left\langle x^{n}, \varphi^{n}\right\rangle=1$. Then $\lambda_{x}=\left\langle T\left(x^{n}\right), \varphi^{n}\right\rangle$. Denote the eigenvalue of $S$ with eigenvector corresponding to $x^{n}$ by $\mu_{x}$. Then

$$
\begin{aligned}
\mu_{x}=\langle & \left.S\left(x^{n}\right), \varphi^{n}\right\rangle \\
& =\left\langle x^{n}, S^{\prime}\left(\varphi^{n}\right)\right\rangle \\
& =\overline{a(\varphi)} \\
& =\overline{\left\langle x^{n}, T^{\prime}\left(\varphi^{n}\right)\right\rangle} \\
& =\overline{\lambda_{x}} .
\end{aligned}
$$

Consider $A=T+S$ in the centraliser of $\widehat{\bigotimes}_{n, s, \epsilon} E$. Then $A\left(x^{n}\right)=2 \mathbf{R e}\left(\lambda_{x}\right) x^{n}$ for all $x$ in $E$. Hence $A^{2}\left(x^{n}\right)=\nu_{x} x^{n}$ with $\nu_{x}$ non-negative for all $x$ in $E$. Letting $b(x)=\left(\nu_{x}\right)^{1 / n}$ we get that

$$
A^{2}\left(x^{n}\right)=(b(x) x)^{n}
$$

for all $x$ in $E$.

Fix $x$ in $E$ for which $A\left(x^{n}\right) \neq 0$. Consider $y$ in $E$ so that $x$ and $y$ are linearly independent for which $b(x) \neq 0$ and $b(y) \neq 0$. This is always possible since $b$ is easily shown to be continuous and for $y$ sufficiently close to $x$ we will have $x^{n}$ and $y^{n}$ linearly independent. Choose $w \in E$ so that $x, y$ and $w$ are linearly independent. Let $E_{o}$ denote the subspace of $E$ spanned by $x, y$ and $w$. By [22, Exercise 10.5.5]) there is a continuous linear mapping $r: E_{o} \rightarrow E_{o}$ such that

$$
A^{2}\left(z^{n}\right)=(r(z))^{n}
$$

for all $z \in E_{o}$. (We need $y$ to avoid the degenerate rank 1 case.) By multiplication by -1 if necessary we may assume that $r(x)=b(x) x$. As in Theorem 3 we get that $b$ is constant on $E_{o}$. As $w$, subject to the constraint that $x, y$ and $w$ being linearly independent, was arbitrary $b$ must be constant on $E$. Thus $A^{2}\left(w^{n}\right)=b w^{n}$ for $w \in E$. Since $\left\{w^{n}: w \in E\right\}$ spans a dense subset of $\widehat{\bigotimes}_{n, s, \epsilon} E$ we see that $A^{2}$ and hence, as in Theorem $3, A$ is a constant multiple of the identity on $\widehat{\bigotimes}_{n, s, \epsilon} E$. Similarly taking $B=i^{-1}(T-S)$ we get that $B$ is a constant multiple of the identity on $\widehat{\bigotimes}_{n, s, \epsilon} E$. From this we conclude that $T$ itself is a constant multiple of the identity and therefore $\widehat{\bigotimes}_{n, s, \epsilon} E$ has trivial centraliser.

The above proof can be easily adapted to cover the case when $\operatorname{dim} E=2$.

As in the real case we get:

Corollary 12 Let $E$ be a complex Banach space and $n$ be a positive integer which is greater than or equal to 2. Suppose that $\overline{\mathcal{E}_{n}\left(E^{\prime \prime}\right)} w^{*}=\overline{\mathcal{E}_{2 n}\left(E^{\prime \prime}\right)} w^{*}$. Then, $Z\left(\mathcal{P}_{A}\left({ }^{n} E\right)\right)$ is trivial. 
Theorem 11 allows a partial extension of Theorem 5 to the complex case.

Theorem 13 Let $E$ be a complex Banach space with the approximation property such that $\overline{\mathcal{E}_{n}\left(E^{\prime \prime}\right)} w^{*}={\overline{\mathcal{E}_{2 n}\left(E^{\prime \prime}\right)}}^{w^{*}}$. Let $n$ be a positive integer and $F$ be a smooth reflexive complex Banach space. Suppose $T: \mathcal{P}_{A}\left({ }^{n} E ; F\right) \rightarrow \mathcal{P}_{A}\left({ }^{n} E ; F\right)$ is an isometry. Then there are isometries $s: E^{\prime} \rightarrow E^{\prime}$ and $S: F \rightarrow F$ such that

$$
T(P)=S\left(\bar{P} \circ s^{\prime} \circ J_{E}\right)
$$

for all $P \in \mathcal{P}_{A}\left({ }^{n} E ; F\right)$.

Proof: Consider an isometry $T: \mathcal{P}_{A}\left({ }^{n} E ; F\right) \rightarrow \mathcal{P}_{A}\left({ }^{n} E ; F\right)$. Then $T$ is an isometry of the underlying real spaces. Applying the argument of Theorem 5 using Theorem 11 instead of Theorem 3 we see that there are real isometries $T_{1}$ of $\mathcal{P}_{A}\left({ }^{n} E\right)$ and $S$ of $F$ such that

$$
T(P \otimes y)=T_{1}(P) \otimes S(y)
$$

for all $P \in \mathcal{P}_{A}\left({ }^{n} E\right)$ and $y \in F$. Given $\lambda \in \mathbf{C}$ we have

$$
T_{1}(\lambda P) \otimes S(y)=T(\lambda P \otimes y)=\lambda T(P \otimes y)=\lambda T_{1}(P) \otimes S(y)
$$

for all $P \in \mathcal{P}_{A}\left({ }^{n} E\right)$ and $y \in F$. This shows that $T_{1}$ is complex linear. A similar argument shows that $S$ is complex linear. Applying [5, Theorem 10] we see that $T_{1}(P)=\bar{P} \circ s^{\prime} \circ J_{E}$ and the result follows.

Theorem 11 allows some insight into centralisers of complex Banach spaces.

A point $x$ is said to be a complex extreme point of the (closed) unit ball of $E$ if $\|x+\lambda y\| \leq 1$ for all $\lambda \in \mathbf{C}$ with $|\lambda|=1$ implies $y=0$. Every real extreme point of $\overline{B_{E}}$ is a complex extreme point. To distinguish between real and complex extreme points we use $\operatorname{Ext}_{\mathbf{C}}(E)$. With these concepts we obtain some important corollaries concerning centralisers of complex Banach spaces.

Theorem 14 Let $E$ be a complex separable Banach space with $\ell_{1} \leftrightarrow E$ and $\operatorname{Ext}_{\mathbf{R}}\left(E^{\prime}\right)=\operatorname{Ext}_{\mathbf{C}}\left(E^{\prime}\right)$ then $E$ has trivial centraliser.

Proof: It follows from the proof of [5, Corollary 11] that $\mathcal{E}_{n}\left(E^{\prime}\right)=\operatorname{Ext}_{\mathbf{R}}\left(E^{\prime}\right)$ for all $n$ and therefore, by Theorem $11, \widehat{\bigotimes}_{n, s, \epsilon} E$ has trivial centralizer. Suppose that $Z(E)$ is non-trivial and take $T \neq k$ Id belonging to $Z(E)$. Then $T \otimes T \otimes \ldots \otimes T$ gives an element of the centraliser of $\widehat{\bigotimes}_{n, s, \epsilon} E$ which is not a constant multiple of the identity contradicting the previous theorem.

We also have

Theorem 15 Let $E$ be a complex reflexive Banach space with $\operatorname{Ext}_{\mathbf{R}}\left(E^{\prime}\right)=$ $\operatorname{Ext}_{\mathbf{C}}\left(E^{\prime}\right)$ then $E$ has trivial centraliser. 
Proof: Proceeding as in [5, Corollary 11] but using [13, Proposition 4.18] instead of the result on $[13$, Page 640$]$ we can show that $\mathcal{E}_{n}\left(E^{\prime}\right)=\operatorname{Ext}_{\mathbf{C}}\left(E^{\prime}\right)$. The remainder of the proof is as in Theorem 14.

In [12, Corollary 2.11] Dineen and Timoney show that a $\mathrm{JBW}^{*}$-triple (a $\mathrm{JB}^{*}$-triple which is also a dual space) has trivial centraliser if and only if it is irreducible. A $\mathrm{JB}^{*}$-triple is irreducible if and only if it cannot be written as a non-trivial $\ell_{\infty}$-sum of its subspaces. If $E$ is a $\mathrm{JB}^{*}$-triple then it follows from $[7,17]$ that $\operatorname{Ext}_{\mathbf{R}}(E)=\operatorname{Ext}_{\mathbf{C}}(E)$. Thus applying Theorem 15 we get

Theorem 16 Let $E$ be a reflexive $J B^{*}$-triple then the centraliser of $E^{\prime}$ is trivial.

We refer the reader to [9] where the reflexive $\mathrm{JB}^{*}$-triples can be found.

\section{The centraliser of $\mathcal{P}\left({ }^{n} E\right)$}

We conclude this paper by examining the centraliser of $\mathcal{P}\left({ }^{n} E\right)$ when $E$ has a Fréchet differentiable norm. We require the notation of strongly and weak*strongly exposed points.

A unit vector $x$ in a Banach space $E$ is strongly exposed if there is a unit vector $\varphi \in E^{\prime}$ so that $\varphi(x)=1$ and given any sequence $\left(x_{k}\right) \subseteq B_{E}$ with $\varphi\left(x_{k}\right) \rightarrow 1$ we can conclude that $x_{k}$ converges to $x$ in norm. We will say that $\varphi$ strongly exposes $B_{E}$ at $x$. When $E=F^{\prime}$ is a dual space and the vector $\varphi$ which strongly exposes $B_{E}$ is in $F$ we shall say that $x$ is weak*-strongly exposed and that $\varphi$ weak $^{*}$-strongly exposes the unit ball of $E$ at $x$.

Theorem 17 Let $E$ be a real or complex reflexive Banach space with a Fréchet differentiable norm and the approximation property and let $n \geq 2$ be a positive integer. Then $\mathcal{P}\left({ }^{n} E^{\prime}\right)$ has trivial centraliser.

Proof: Since $E$ has a Fréchet differentiable norm $E^{\prime}$ has Radon-NikodýmProperty. It therefore follows from [6, Proposition 3] (see also [8, Theorem 1.5]) that $\mathcal{P}_{I}\left({ }^{n} E\right)$ is isometrically isomorphic to $\mathcal{P}_{N}\left({ }^{n} E\right)$. As $E^{\prime}$ has the approximation property it follows that $\mathcal{P}\left({ }^{n} E^{\prime}\right)$ is the bidual of $\widehat{\bigotimes}_{n, s, \epsilon} E$. Further, as the norm on $E$ is Fréchet differentiable, it follows from [6, Corollary 15] that the set of strongly exposed points of the unit ball of $\mathcal{P}_{I}\left({ }^{n} E\right)$ is equal to the set $\left\{ \pm \varphi^{n}: \varphi \in E^{\prime},\|\varphi\|=1\right\}$. In particular, the sets of extreme and strongly exposed points of the unit ball of $\mathcal{P}_{I}\left({ }^{n} E\right)$ are equal. It follows that the set of weak*-strongly exposed points of the unit ball of $\mathcal{P}\left({ }^{n} E^{\prime}\right)^{\prime}$ is equal to $\left\{ \pm \varphi^{n}: \varphi \in E^{\prime},\|\varphi\|=1\right\}$ (see the comment at the end of [20, Theorem 5.2.1]). Let $T$ be in the centraliser of $\mathcal{P}\left({ }^{n} E^{\prime}\right)$. Then, given $\varphi$ in $E^{\prime}, \varphi^{n}$ is a weak*strongly exposed point of the unit ball of $\mathcal{P}\left({ }^{n} E^{\prime}\right)^{\prime}$. In particular, it is an extreme point of the unit ball of $\mathcal{P}\left({ }^{n} E^{\prime}\right)^{\prime}$. Hence, we have that

$$
T^{\prime}\left(\varphi^{n}\right)=a(\varphi) \varphi^{n}
$$

for some scalar $a(\varphi)$ and each $\varphi$ in $E^{\prime}$. Thus $\left.T^{\prime}\right|_{\mathcal{P}_{I}\left({ }^{n} E\right)}$ maps $\mathcal{P}_{I}\left({ }^{n} E\right)$ onto $\mathcal{P}_{I}\left({ }^{n} E\right)$ and hence $T$ is the transpose of an operator $S$ from $\mathcal{P}_{I}\left({ }^{n} E^{\prime}\right)$ into $\mathcal{P}_{I}\left({ }^{n} E^{\prime}\right)$ (see the 
proof of [18, Observación 3.8]). Thus $T^{\prime}=\left(\left.T^{\prime}\right|_{\left.\mathcal{P}_{I}{ }^{n} E\right)}\right)^{\prime \prime}$. The argument given in Theorem 3 or Theorem 11 implies that $\left.T^{\prime}\right|_{\mathcal{P}_{I}\left({ }^{n} E\right)}$ and hence $T^{\prime}=\left(\left.T^{\prime}\right|_{\mathcal{P}_{I}\left({ }^{n} E\right)}\right)^{\prime \prime}$ is a constant multiple of the identity proving that $\mathcal{P}\left({ }^{n} E^{\prime}\right)$ has trivial centraliser.

In particular, we see that $\mathcal{P}\left({ }^{n} L^{p}(\mu)\right)$ and $\mathcal{P}\left({ }^{n} \ell_{p}\right)$ have trivial centralisers for $1<p<\infty$. Since $\mathcal{B}(H)$, the space continuous linear operators from a Hilbert space $H$ into $H$, is isometrically isomorphic to $\mathcal{P}\left({ }^{2} H\right)$ we recover the result that $\mathcal{B}(H)$ has trivial centraliser. There are many ways to see this. Perhaps the easiest is to observe that $\mathcal{B}(H)$ is a unital $\mathrm{C}^{*}$-algebra and therefore its centraliser is $\left\{M_{T}: T\right.$ is in the centre of $\left.\mathcal{B}(H)\right\}$. Since $\mathcal{B}(H)$ has trivial centre the result follows. In addition, spaces which satisfy the conditions of Theorem 17 have the strong Banach-Stone property.

A projection $P$ on a Banach space $E$ is said to be an $L$-projection if

$$
\|x\|=\|P(x)\|+\|(I-P)(x)\|
$$

for all $x$ in $E$. Using [14, Theorem I.3.14 (b)] we obtain the following corollary to Theorem 17.

Corollary 18 Let $E$ be a real or complex reflexive Banach space with a Fréchet differentiable norm and the approximation property and let $n \geq 2$ be a positive integer. Then $\mathcal{P}_{I}\left({ }^{n} E\right)$ contains no non-trivial L-projections.

\section{Acknowldegements}

The second author is grateful to the Department of Mathematics, University College Dublin, for its hospitality during her visit of April-May 2005 when this paper was written.

\section{References}

[1] Alfsen E.M \& Effros E.G. Structure in real Banach spaces, parts I and II, Ann. Math., 96, (1972), 98-128 and 129-173.

[2] Aron R.M. \& Berner P., A Hahn-Banach extension theorem for analytic mappings, Bull. Math. Soc. France, 106, (1978), 3-24.

[3] Behrends E., M-structure and the Banach-Stone Theorem, Lecture Motes in Mathematics, $\mathbf{7 3 6},(1979)$.

[4] Boyd C., Dineen S. \& Rueda P. Weakly uniformly continuous holomorphic functions and the approximation property, Indag. Math., N.S., 12, (2), (2001), 147-156.

[5] Boyd C. \& LAssalle S., Isometries between spaces of homogeneous polynomials, J. Functional Analysis, 224, (2), (2005), 281-295. 
[6] Boyd C. \& Ryan R.A., Geometric theory of integral polynomials and symmetric tensor products, J. Functional Analysis, 179, (2001), 18-42.

[7] Braun R., Kaup W. \& Upmeier H., A holomorphic characterization of Jordan $\mathrm{C}^{*}$-algebras, Math. Zeit., 161, (1978), 277-290.

[8] Carando D. \& Dimant V., Duality of spaces of nuclear and integral polynomials, J. Math. Anal. Appl., 241, (2000), 107-121.

[9] Chu, C.-H. \& Iоснuм B., Weakly compact operators on Jordan triples, Math. Ann., 281, (1988), 451-458.

[10] Dineen S., Complex analysis on infinite dimensional spaces, Monographs in Mathematics, Springer-Verlag, (1999).

[11] Dineen S., Holomorphic types on Banach space, Studia Math., 39, (1971), 241-288.

[12] Dineen S. \& Timoney R. The centroid of a JB*-triple system, Math. Scand. 62, (1988), 327-342.

[13] Fonf, V.P., Lindenstrauss J. \& Phelps, R.R., Infinite dimensional convexity, Handbook of the Geometry of Banach spaces, Edited by W. B. Johnson and J. Lindenstrauss, (2001), Elsevier Science B.V., 599-670.

[14] Harmand P., Werner D. \& Werner W., M-Ideals in Banach spaces and Banach Algebras, Lecture Notes in Mathematics, 1547, (1993).

[15] Holmes R.B., Geometric Functional Analysis and its Applications, Springer-Verlag Graduate Texts in Math., 24, (1975).

[16] Jarosz K., Isometries between injective tensor products of Banach spaces, Pacific J. Math., 121, (2), (1986), 383-396.

[17] Kaup W. \& Upmeier H., Jordan algebras and symmetric Siegel domains in Banach spaces, Math. Zeit., 157, (1977), 179-200.

[18] Lassalle S., Polinomios sobre un espacio de Banach y su relación con el dual, Ph.D. Thesis, Universidad de Buenos Aires, (2001).

[19] Lassalle S. \& Zalduendo I., To what extent does the dual Banach space $E^{\prime}$ determine the polynomials over E? Ark. Mat., 38, (2000), 343-354.

[20] Ruess W.M, Duality and geometry of spaces of compact operators, Functional Analysis, surveys and recent results, III, Paderborn, 1983, NorthHolland Math. Studies, 90, (1984), 59-78.

[21] Ruess W.M. \& Stegall C.P., Exposed and denting points in the duals of operator spaces, Israel J. Math., 53, (1986), 163-190.

[22] Shaw R., Linear Algebra and Group Representation, Volume II, Academic Press, (1983). 
[23] Werner W., The centraliser of the injective tensor product, Bull. Austral. Math. Soc., 44, (1991), 357-365.

[24] Wickstead A.W., The centraliser of $E \otimes_{\lambda} F$, Pacific J. Math., 65, (1976), $563-571$.

Department of Mathematics, University College Dublin, Belfield, Dublin 4, Ireland.

e-mail: Christopher.Boyd@ucd.ie

Departamento de Matemática, Pab. I - Cuidad Universitaria, (FCEN), Universidad de Buenos Aires, (1428) Buenos Aires, Argentina.

e-mail: slassall@dm.uba.ar 